A - Research concept and design

B - Collection and/or assembly of data

$\mathrm{C}$ - Data analysis and interpretation

D - Writing the article

E - Critical revision of the article

F - Final approval of article

Received: 2021-06-22 Accepted: 2021-10-13 Published: 2021-10-15

\section{Sleep disturbances and functional independence improvement in a population of individuals hospitalized in intensive rehabilitation units: observational study}

Giovanni Galeoto*1,2,A,C,E-F (D), Anna Berardi ${ }^{1, A, C-D}$ (D), Maria Letizia Grassi ${ }^{3, B, D}$ (D) Alessia Scialpi $i^{3, B, D}$ iD, Donatella Valente $^{1, E-F}$ (D), Marco Tofani ${ }^{4, A, E}$ (D), Marco Paoloni $i^{5, A, F}$ (D)

${ }^{1}$ Department Human Neurosciences, Sapienza University of Rome, Italy ${ }^{2}$ IRCSS Neuromed, Pozzilli, IS, Molise, Italy

${ }^{3}$ Sapienza University of Rome, Italy

${ }^{4}$ Department of Public Health and Infection disease, Sapienza University of Rome, Italy

${ }^{5}$ Department of Anatomical, Histological, Forensic and Orthopaedic Sciences, Sapienza University of Rome, Italy

${ }^{*}$ Correspondence: Giovanni Galeoto; Department Human Neurosciences, Sapienza University of Rome, Italy; email: giovanni.galeoto@uniroma1.it

\begin{abstract}
Introduction: Quality of sleep in hospitals is an important issue, especially for rehabilitation patients. The aim of this study was to evaluate the development of sleep disturbances in a population of individuals hospitalized in intensive rehabilitation units and to investigate relationship with functional independence.

Material and methods: The sample was evaluated with the General Sleep Disturbance Scale (GSDS-IT) and Bartel Index (IcaBI) three times: at admission ( $\mathrm{t} 0$ ), seven days after admission ( $\mathrm{t} 1$ ), and 25 days after admission ( $\mathrm{t} 2$ ). The variation in sleep quality between t0-t1-t 2 was calculated by performing a paired samples t-test between the scores' averages. A comparison of the functional independence level evaluated thorugh IcaBI of subjects with and without sleep disorders according to GSDS-IT cut-off points was performed via an independent samples t-test between the averages of the scores obtained on the IcaBI at times t0 and $\mathrm{t} 2$.

Results: 138 individuals were included in the study, 77 of whom completed the evaluation at 2 . Comparison of the mean scores on the GSDS-IT showed a statistically significant worsening of the quality of sleep between t0-t1-t2 $(p<0.01)$. Comparison of the mean scores on the IcaBI showed a statistically significant improvement in functional independence between $\mathrm{t} 0-\mathrm{t} 1-\mathrm{t} 2(\mathrm{p}<0.01)$. The results showed no significant difference between those with or without sleep disorders in terms of improvement of IcaBI scores after 25 days of hospitalization.

Conclusions: This study represent a starting point in highlighting the development of sleep disturbances during hospitalization in rehabilitation units, raising awareness about the importance of adequate sleep among vulnerable hospital population.
\end{abstract}

Keywords: hospitalization, rehabilitation, sleep disorder

\section{Introduction}

Sleep disturbance and poor sleep quality is associated with a general negative impact on health, such as increased health care costs and increased risk of mental disorders [1-4]. In this study, sleep disturbance was defined as either sleep deprivation resulting from an inadequate total amount of sleep or sleep

This is an Open Access journal, all articles are distributed under the terms of the Creative Commons Attribution-NonCommercial-ShareAlike 4.0 International (CC BY-NC-SA 4.0). License (http://creativecommons. org/licenses/by-nc-sa/4.0/). 
disruption resulting from fragmented sleep during the night [5-8]. Hospitalization is a major cause of sleeping pattern disturbance, because of the effects of illness, drug therapies, pain, and depression, caregiver interruptions and environmental noise $[9,10]$. In fact, the hospital environment is characterized by noise from different sources such as parametric monitoring devices that indicate a patient's health condition with noisy alarms and "beeps" [11]. The negative implications for the sleep quality [12], can lead in turn hinder recovery from pathologies $[8,11]$. It has previously been shown that daytime sleepiness is a predictor of poor functional recovery in hospitalized geriatric patients: a significantly positive correlation between the severity of daytime sleepiness and the worsening of the Barthel Index was identified [12]. It is therefore evident that medical awareness of the impact of sleep disorders on hospitalized patients is of vital importance, and that knowledge of the major factors that disturb sleep during hospitalization will allow both researchers and hospital staff to systematically evaluate and treat sleep issues. Although inadequate sleep has a demonstrated negative association with health care outcomes, to date, few studies have examined sleep in intensive rehabilitation units or its association with functional independence outcomes [13-15]. Quality of sleep in hospitals is an important issue in general but possibly even more so for rehabilitation patients who may have long stays. In this study, sleep disturbances were analyzed among patients hospitalized in intensive rehabilitation units, which are characterized by intensive evaluative and therapeutic interventions. Such interventions are typically directed toward patients in the post-acute phase, when rehabilitation can positively influence functional recovery by managing and/or reducing the extent of impairment, i.e., when disability is most modifiable; this phase may also be necessary in situations in which the pathological event is exacerbated or reoccurring. Hospitalization in an intensive rehabilitation unit is usually 25 days. The administration on the day of admission to the hospital of the General Sleep Disturbance Scale (GSDS), that measures sleep disturbances experienced during the previous week, made it possible to assess the quality of the patient's sleep in daily life prior to admission. Furthermore, the time frame of the scale coincides with the period in which factors that could alter the quality of sleep, such as agitation and fear, can more easily occur.

Thus, the aim of this study was to evaluate the presence and development of sleep disturbances in a population of individuals hospitalized in intensive rehabilitation units and to investigate its relationship with functional independence.

\section{Materials and methods}

It was observational study using interrupted time series.

\section{Participants}

Participants were recruited from the Department of Physical Rehabilitation Medicine at the "Policlinico Umberto I" University Hospital and at the "Policlinico Italia" hospital in Rome.

To be included, participants had to meet the following criteria:

1. be admitted to an intensive rehabilitation unit;

2. have not been an inpatient for longer than seven days at the time of recruitment;

3 . be at least 18 years old;

4. have no insomnia symptoms as defined by Diagnostic and Statistical Manual of Mental Disorders (DSM-V) [16] diagnostic criteria [17], the tool used for this was The Sleep Condition Indicator [18];

5. have the ability to communicate in Italian;

6. give signed informed consent for the processing of personal data.

Patients admitted to the intensive rehabilitation unit were informed of the purpose of the study, as well of the methods and timing of the administration of the instruments. Those who showed interest in the study were assessed against the inclusion and DSM-V diagnostic criteria, eligible participants provided their data and signed the informed consent form $[19,20]$. Institutional ethics committee approval was not required for this stu$\mathrm{dy}$, this research involved secondary use of clinical data which were provided without any identifier or group of identifiers which would allow attribution of private information to an individual. The Sapienza University Ethics Committee approved the research conducted to collect the data (Protocol NumberEQL2-15-01, Approval No. 3997).

The GSDS [5] is a self-assessment scale which evaluates how often in the previous week sleep-related disturbances occurred. The total score ranges from 0 to 147 , higher scores indicating a higher frequency of sleep disturbances. In a previous study among the healthy Italian population, the optimal cut-off point, i.e., distinguishing good from bad sleep [21,22], for the GSDS was 38.5. In this study the Italian version of the scale was used - GSDS-IT. The Barthel Index [23] is a tool widely used in hospitals to measure independence in activities of daily living (ADLs). The total score ranges from 0 to 100 [24].

The GSDS-IT was administered face-to-face by three rehabilitation experts (two occupational therapists and one physiotherapist) at three time points: at admission to the intensive rehabilitation unit (t0) to detect 
the presence of any sleep disturbances during the week prior to admission; seven days after admission (t1) to detect the presence of any sleep disturbances arising during the first week of admission, or to detect the worsening or persistence of disorders already present before admission; and 25 days from admission (t2) to detect an increase, decrease, or persistence of such disorders. Concurrently with the GSDS-IT [22], participants were given the Italian version of the Barthel Index (IcaBI) [24] to evaluate the functional recovery. Demographic and diagnostic information were also collected from participants. The presence or absence of sleep disturbances at admission ( $\mathrm{t} 0$ ) was assessed using the GSDSIT cut-off score calculated during the validation of the scale among the healthy Italian population [22].

\section{Statistical analysis}

All statistical analyses were performed using IBMSPSS version 23.00. In order to assess changes in sleep quality during hospitalization, the average of the scores obtained by the GSDS-IT at time t0 was compared with the mean obtained by the same subjects at $t 1$ and $t 2$, separately, through a paired samples t-test. Differences in the functional independence level between participants showing or not showing sleep disorders, according to the GSDS-IT cut-off score, were assessed. For this purpose, the mean scores of the IcaBI [25] of the two groups were compared by independent samples t-tests. Since it was not possible to record data at time $t 2$ for some participants, all statistical analyses at time $\mathrm{t} 2$ were performed only on the sub-group of subjects for which data could be recorded. The significance level was set for $\mathrm{p} \leq 0.05$.

\section{Results}

Initially, 184 patients from two intensive rehabilitation units in Rome were recruited. Of these individuals, 138 signed the informed consent form and were therefore included in the study. Table 1 reports the demographic characteristics of 138 participants evaluated at $t 0$.

Figure 1 shows participants' recruitment and inclusion in the study.

An independent t-test showed no statistical differences between participants with or without sleep disturbances as detected according to the GSDS cut-off points.

138 participants were evaluated with the GSDS-IT [21] upon admission ( $\mathrm{t} 0), 64$ of whom (46.38\%) tested positive for sleep disturbances according to the cut-off score obtained during the validation of the scale among the healthy Italian population [21]. One week after admission (t1), 108 participants tested positive for sleep disturbances $(78.26 \%,+31.88 \%$ with respect to $\mathrm{t} 0$ ). After 25 days from admission ( $\mathrm{t} 2$ ), the number of participants for whom it was possible to record data decreased from 138 to 77 . Of these, $63(81.81 \%,+3.55 \%$ compared to t1) showed signs of sleep disturbances according to the GSDS cut-off points. A comparison

Tab. 1. Demographic characteristics of the 138 participants at admission to the intensive rehabilitation unit

\begin{tabular}{lccc}
\hline & All sample & Without sleep disturbance* & With sleep disturbance* \\
& $\mathrm{N}=138$ & $\mathrm{~N}=74$ & $\mathrm{~N}=64$ \\
\hline Mean age $\pm \mathrm{SD}$ & $70.4 \pm 12.41$ & $70.1 \pm 14.12$ & $70.7 \pm 10.18$ \\
Females number (\%) & $47(34.1)$ & $46(62.2)$ & $46(71.9)$ \\
Occupation number (\%) & & & 0.80 \\
Employee & $26(18.8)$ & $16(21.6)$ & $10(15.6)$ \\
Private job & $12(8.7)$ & $6(8.1)$ & $6(9.4)$ \\
Retired & $100(72.5)$ & $52(70.3)$ & $48(75)$ \\
Family number $(\%)$ & & & $41(64.1)$ \\
Married & $76(55.1)$ & $35(47.3)$ & $10(15.6)$ \\
Single & $33(23.9)$ & $23(31.1)$ & $13(20.3)$ \\
Widow & $29(21)$ & $16(21.6)$ & $40.22 \pm 6$ \\
Barthel Index mean \pm SD & $40.2 \pm 5.9$ & $40.19 \pm 5.8$ & \\
\hline
\end{tabular}

\footnotetext{
* according to GSDS cut-off points 38.5.
} 


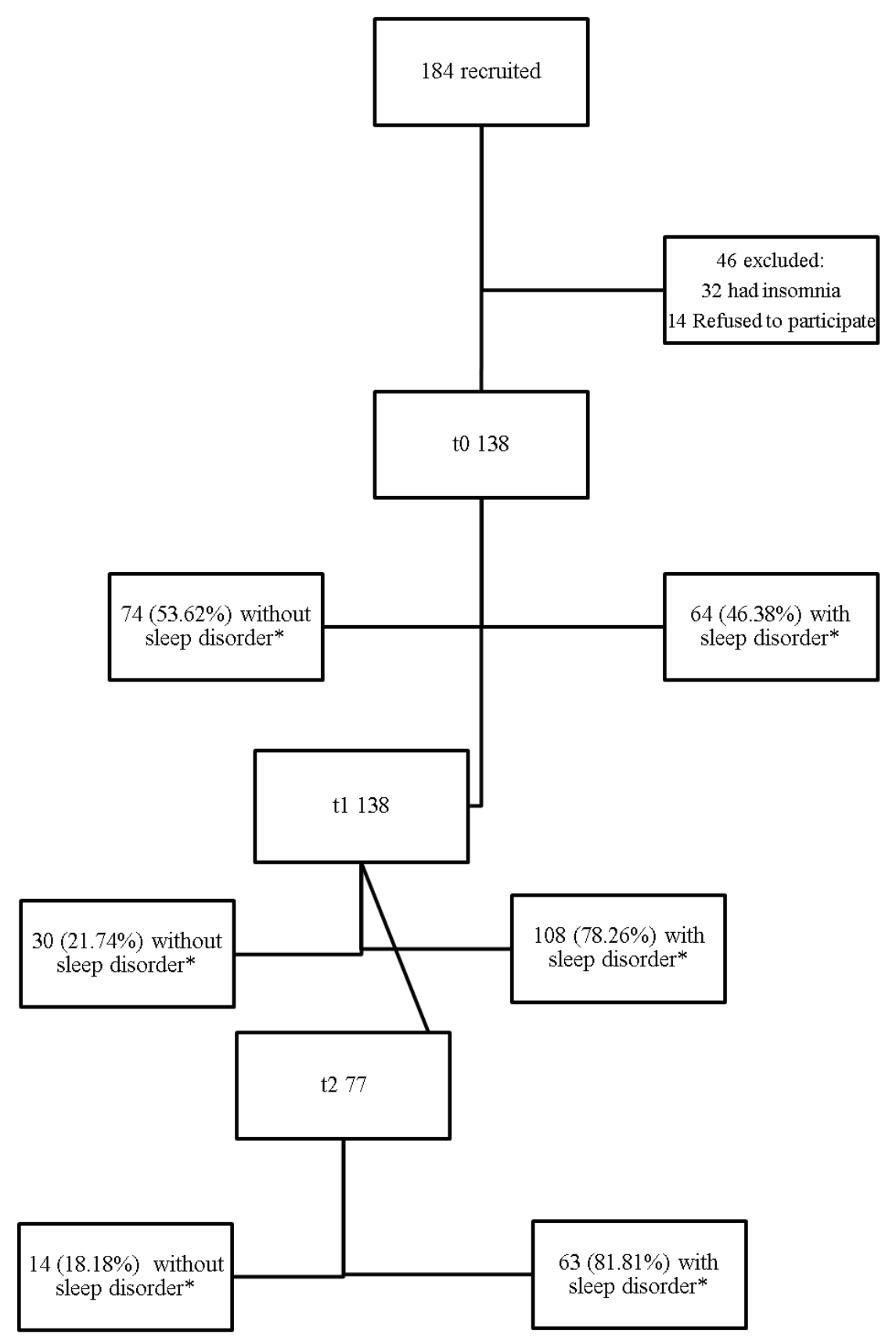

Fig. 1. Flowchart of recruitment and inclusion of participants

between the averages of scores on the GSDS-IT showed a statistically significant increase in the total score as well as in the scores of all sub-scales except the fifth between times $\mathrm{t} 0$ and $\mathrm{t} 2$, as reported in Table 2. However, a comparison between the averages of the scores on the GSDS-IT recorded at $\mathrm{t} 1$ and $\mathrm{t} 2$ showed a decrease in the score, thus indicating an improvement in sleep quality from $t 1$ to $t 2$, as reported in Table 2 .

A comparison of the IcaBI scores between time t0 and $\mathrm{t} 1$ showed a statistically significant improvement in the functional independence of the 138 participants one week after admission, despite the occurrence of sleep disturbances, as reported in Table 3.

At $t 1$, a comparison of the IcaBI scores between those with $(\mathrm{n}=108)$ and without $(\mathrm{n}=30)$ sleep disturbances did not show a statistically significant difference between the two groups, although there was a worsening in sleep quality and duration, as reported in Table 2 . The same comparison was repeated at $\mathrm{t} 2$; again, no statistically significant difference was found in the functional independence level between participants with and without sleep disturbances, as shown in Table 4. 
Tab. 2. Comparison between the averages of scores on the GSDS-IT in the total score as well as in the scores of all sub-scales between times $\mathrm{t} 0$ and $\mathrm{t} 2$

\begin{tabular}{lccrc}
\hline GSDS & $\begin{array}{c}\text { All sample (77) T0 } \\
\text { mean } \pm \text { SD }\end{array}$ & $\begin{array}{c}\text { All sample (77) T1 } \\
\text { mean } \pm \text { SD }\end{array}$ & All sample (77) T2 & p t0-t2 \\
\hline Initial insomnia & $1.78 \pm 2.75$ & $3.83 \pm 3.24$ & $3.65 \pm 3.26$ & $<0.001$ \\
Maintenance insomnia & $3.06 \pm 2.51$ & $4.65 \pm 2.22$ & $5.13 \pm 2.14$ & $<0.001$ \\
Quality of sleep & $0.46 \pm 1.76$ & $2.05 \pm 2.07$ & $1.81 \pm 2.06$ & $<0.001$ \\
Quantity of sleep & $1.12 \pm 1.54$ & $1.97 \pm 1.70$ & $1.82 \pm 1.69$ & $<0.01$ \\
Daytime functionality & $2.50 \pm 1.02$ & $2.75 \pm 1.35$ & $2.62 \pm 1.17$ & 0.488 \\
Use of substances that help & $0.30 \pm 0.60$ & $0.87 \pm 0.80$ & $0.73 \pm 0.76$ & $<0.001$ \\
induce sleep & $39.07 \pm 12.09$ & $53.48 \pm 15.71$ & $49.86 \pm 13.30$ & $<0.001$ \\
\hline Total & & & & \\
\hline
\end{tabular}

Tab. 3. Difference of Barthel index score between t0 (admission to the intensive rehabilitation unit) and t1 (7 days after admission) assessed through t-test for paired samples. Differences at $\mathrm{t} 2$ between subsamples of people with or without sleep disturbance assessed through t-test for independent samples

\begin{tabular}{lcccccc}
\hline & $\begin{array}{c}\text { All sample (138) T0 } \\
\text { mean } \pm \text { SD }\end{array}$ & $\begin{array}{c}\text { All sample (138) T1 } \\
\text { mean } \pm \text { SD }\end{array}$ & P & $\begin{array}{c}\text { Without sleep } \\
\text { disturbance* (28) T1 } \\
\text { mean } \pm \text { SD }\end{array}$ & $\begin{array}{c}\text { With sleep } \\
\text { disturbance* T1 (110) } \\
\text { mean } \pm \text { SD }\end{array}$ & p \\
\hline Feeding & $9.43 \pm 1.55$ & $9.78 \pm 0.85$ & $<0.01$ & $10 \pm 0$ & $9.72 \pm 0.95$ & 0.12 \\
Bathing & $0.82 \pm 1.09$ & $3.57 \pm 1.59$ & $<0.001$ & $3.82 \pm 1.63$ & $3.50 \pm 1.58$ & 0.34 \\
Grooming & $1.55 \pm 1.08$ & $4.68 \pm 1.01$ & $<0.001$ & $4.64 \pm 1.10$ & $4.69 \pm 0.99$ & 0.82 \\
Dressing & $3.38 \pm 1.76$ & $7.62 \pm 2.57$ & $<0.001$ & $7.54 \pm 2.50$ & $7.65 \pm 2.59$ & 0.84 \\
Bowel & $9.53 \pm 1.25$ & $9.64 \pm 1.41$ & 0.52 & $9.50 \pm 1.93$ & $9.67 \pm 1.25$ & 0.56 \\
Bladder & $8.61 \pm 2.58$ & $9.33 \pm 1.95$ & $<0.01$ & $8.86 \pm 3.15$ & $9.45 \pm 1.50$ & 0.15 \\
Toilet use & $1.98 \pm 1.90$ & $8 \pm 3.56$ & $<0.001$ & $7.86 \pm 3.55$ & $8.04 \pm 3.58$ & 0.81 \\
Transfer & $3.25 \pm 1.78$ & $12.38 \pm 4.50$ & $<0.001$ & $12.57 \pm 4.11$ & $12.33 \pm 4.60$ & 0.80 \\
Mobility & $0.60 \pm 1.34$ & $9.36 \pm 6.39$ & $<0.001$ & $8.46 \pm 6.83$ & $9.59 \pm 6.28$ & 0.41 \\
Stairs & $0.04 \pm 0.30$ & $1.51 \pm 3.19$ & $<0.001$ & $0.71 \pm 1.96$ & $1.71 \pm 3.41$ & 0.14 \\
Wheelchair use & $1.14 \pm 1.47$ & $0.80 \pm 1.80$ & 0.10 & $0.89 \pm 1.95$ & $0.77 \pm 1.77$ & 0.75 \\
\hline Total & $40.31 \pm 5.88$ & $76.66 \pm 19.15$ & $<0.001$ & $74.86 \pm 18.17$ & $77.12 \pm 19.45$ & 0.58 \\
\hline
\end{tabular}

* sleep disturbance determined according to GSDS cut off points.

\section{Discussion}

The aim of this observational study, using interrupted time series, was to evaluate the presence and development of sleep disturbances in a population of individuals hospitalized in intensive rehabilitation units and to investigate relationship with functional independence. The GSDS-IT and the modified IcaBI have been administered at admission, seven days, and 25 days.

Among the study participants who were hospitalized without insomnia according to the DSM-V diagnostic criteria, [17] that forty-six \% of patients had sleep disturbances symptoms (scores above 38.5 on the GSDS) in the week prior to admission, and $82 \%$ of the population had developed these symptoms by 25 days. The IcaBI significantly improved during admission, but there was no difference in scores when those with/without 'insomnia' symptoms were compared. Thus, the development of sleep disturbances did not seem to significantly affect improvement to functional independence as measured by the IcaBI. This result is understandable given that during the first week of admission, all 
Tab. 4. Difference of Barthel index score between t0 (admission to the intensive rehabilitation unit) and $\mathrm{t} 2$ ( 25 days after admission) assessed through t-test for paired samples. Differences at $t 2$ between subsamples of people with or without sleep disturbance assessed through t-test for independent samples

\begin{tabular}{lcccccc}
\hline & $\begin{array}{c}\text { All sample (77) T0 } \\
\text { mean } \pm \text { SD }\end{array}$ & All sample (77) T2 & P & $\begin{array}{c}\text { Without sleep } \\
\text { disturbance* (14) T2 }\end{array}$ & $\begin{array}{c}\text { With sleep } \\
\text { disturbance* (63) T2 }\end{array}$ & P \\
\hline Feeding & $9.65 \pm 0.89$ & $10 \pm 0$ & $<0.001$ & $10 \pm 0$ & $10 \pm 0$ & \\
Bathing & $0.78 \pm 1.20$ & $4.35 \pm 1.13$ & $<0.001$ & $4.56 \pm 0.16$ & $4.30 \pm 0.16$ & 0.404 \\
Grooming & $1.47 \pm 1.10$ & $4.88 \pm 0.56$ & $<0.001$ & $4.88 \pm 0.13$ & $4.89 \pm 0.07$ & 0.949 \\
Dressing & $3.16 \pm 1.69$ & $8.82 \pm 2.00$ & $<0.001$ & $9.44 \pm 0.34$ & $8.66 \pm 0.27$ & 0.167 \\
Bowel & $9.60 \pm 1.21$ & $9.95 \pm 0.32$ & $<0.01$ & $10 \pm 0$ & $9.93 \pm 0.05$ & 0.470 \\
Bladder & $8.81 \pm 2.36$ & $9.74 \pm 1.04$ & $<0.001$ & $10 \pm 0$ & 9.67 & 0.266 \\
Toilet use & $1.84 \pm 2.01$ & $9.49 \pm 1.92$ & $<0.001$ & $9.38 \pm 0.63$ & 9.52 & 0.784 \\
Transfer & $3.12 \pm 1.70$ & $14.36 \pm 2.50$ & $<0.001$ & $14.25 \pm 0.75$ & 14.39 & 0.838 \\
Mobility & $0.35 \pm 0.97$ & $13.47 \pm 3.93$ & $<0.001$ & $13.63 \pm 0.85$ & 13.43 & 0.858 \\
Stairs & $0.04 \pm 0.34$ & $4.97 \pm 4.30$ & $<0.001$ & $4.38 \pm 1.04$ & 5.13 & 0.535 \\
Wheelchair use & $1.12 \pm 1.36$ & $2.70 \pm 2.49$ & $<0.001$ & $1.56 \pm 0.60$ & 2.95 & 0.046 \\
\hline Total & $39.87 \pm 5.81$ & $92.70 \pm 13.50$ & $<0.001$ & $92.06 \pm 3.14$ & 92.87 & 0.833 \\
\hline
\end{tabular}

* sleep disturbance determined according to GSDS cut off points.

participants performed rehabilitative treatments aimed at improving their physical and functional performance. The improvement in functional independence one week after admission was comparable between individuals who did not experience sleep disturbances upon hospitalization and those who did. This may be due to the reduced time frame in which these disorders arose, which may not have been enough to interfere with the functional plan. Twenty-five days after admission, most of the participants were still experiencing sleep disturbances associated with hospitalization. However, the functional independence of the participants continued to improve regardless of the presence or absence of sleep disturbances. Although slightly higher improvement was observed among those participants not experiencing a sleep disturbance, the difference was not statistically significant.

However, accordingly to the above results also Hoch et al. [27] could not demonstrate that sleepiness had an impact on functional status, when younger and older healthy subjects were compared. Of note, in that small study self-reported sleepiness was rather mild. In contrast to this, some researches has investigated sleep disturbance/deprivation and the impact on cognition and/or function on elderly population [12]. Stenholm et al. [28] demonstrated that excessive tiredness influenced measured and self-reported mobility outcomes in participants. Gooneratne et al. [29] found that in elderly subjects ( $>65$ years) functional status was significantly impaired in subjects with daytime sleepiness. Even though sleep deprivation showed adverse effects of on recovery from illness, sleep deprivation among participants remains an incompletely addressed problem [27-29]. Due to the side effects of sedative hypnotics, in previous studies behavioral interventions are recommended as a first-line therapy prior to using pharmacologic therapy [13,30-32] Environmental modifications including designated quiet time and ear plugs/eye masks, are one of the most recommended intervention, as well as relaxation techniques, improved sleep hygiene or reduced sleep interruption programs, and daytime bright light therapy [14,30-32].

\section{Limitation of the study}

Although the IcaBI scale was chosen because it is commonly used to assess participants' level of independence in intensive rehabilitation units in Italy, its results may be too generic for examining associations in functional improvement between people with or without sleep disturbances. Future studies could use more specific scales, such as Berg Balance Score (BBS), or physical activity scale for the elderly (PASE), to obtain more detailed information about the impact of sleep disturbances on rehabilitation outcomes. Moreover, the proportion of patient who did not have difficulties with sleep was so small that it would be difficult to determine if sleep had an impact or not on IcaBI. The percentage of people with sleep disorder was $80 \%$ by one week after admission. It is important to be clear in saying that this was a dichotomy around a cut-off point on a scale 
i.e. they could be one point over the cut off or 10 points over. Therefore, severity of sleep disorder is not a variable authors looked at. Another limitation is that further information about the study population such as known factors that are related to poor sleep, including pain/depression/anxiety as well as change of environment/bed 'first night effect' (for first week), pain, mental health, medical conditions, medications, side rooms/bayswere not collected.

\section{Conclusions}

There is a need to highlight the need for rehabilitation treatments focused on sleep disturbances in combination with the pharmacological treatments already in use in the hospital. This study demonstrated that the duration and quality of sleep among participants in intensive rehabilitation units were both significantly affected, raising awareness about the importance of adequate sleep among vulnerable hospital populations and introducing interventions to target sleep-disturbing factors may therefore improve healing [8]. This study represent a starting point in highlighting the development of sleep disturbances during hospitalization in intensive rehabilitation units. Future studies should deepen this analysis finding the exact cause to thereby guide treatment(s).

\section{Funding}

This research received no external funding.

\section{Conflict of interests}

The authors have no conflict of interest to declare.

\section{References}

1. Hirshkowitz M. Normal human sleep: An overview. Med Clin North Am 2004; 88(3): 551-65.

2. American Academy of Sleep Medicine. International Classification of Sleep Disorders: Diagnostic and Coding Manual. 3rd ed. 2014.

3. Hirshkowitz M, Whiton K, Albert SM, Alessi C, Bruni O, DonCarlos L, et al. National sleep foundation's sleep time duration recommendations: Methodology and results summary. Sleep Heal 2015; 1(1): 40-43.

4. Kryger MH, Roth T, Dement WC. Principles and Practice of Sleep Medicine: Fifth Edition. 2010.

5. Lee KA. Self-reported sleep disturbances in employed women. Sleep 1992; 15(6): 493-8.

6. Dobing S, Frolova N, McAlister F, Ringrose J. Sleep quality and factors influencing self-reported sleep duration and quality in the general internal medicine inpatient population. PLoS One 2016; 11(6): e0156735.
7. Rahe C, Czira ME, Teismann H, Berger K. Associations between poor sleep quality and different measures of obesity. Sleep Med 2015; 16(10): 1225-8.

8. Wesselius HM, Van Den Ende ES, Alsma J, Ter Maaten JC, Schuit SCE, Stassen PM, et al. Quality and quantity of sleep and factors associated with sleep disturbance in hospitalized patients. JAMA Intern Med 2018; 178(9): 1201-1208.

9. Sasai T, Inoue Y, Komada Y, Nomura T, Matsuura M, Matsushima E. Effects of insomnia and sleep medication on health-related quality of life. Sleep Med 2010; 11(5): 452-7.

10. Frighetto L, Marra C, Bandali S, Wilbur K, Naumann T, Jewesson P. An assessment of quality of sleep and the use drugs with sedating properties in hospitalized adult patients. Health Qual Life Outcomes 2004; 2: 17 .

11. Young JS, Bougeois JA, Hilty DM, Hardin KA. Sleep in hospitalized medical patients, Part 1: Factors affecting sleep. J Hosp Med 2008; 3(6): 473-82.

12. Frohnhofen H, Popp R, Frohnhofen K, Fulda S. Impact of daytime sleepiness on rehabilitation outcome in the elderly. Adv. Exp. Med. Biol., 2013; 755: 103-10.

13. Lewandowska K, Małkiewicz MA, Siemiński M, Cubała WJ, Winklewski PJ, Mędrzycka-Dąbrowska WA. The role of melatonin and melatonin receptor agonist in the prevention of sleep disturbances and delirium in intensive care unit - a clinical review. Sleep Med 2020; 69: 127-34.

14. Lewandowska K, Mędrzycka-Dąbrowska W, KwiecieńJaguś K, Czyż-Szypenbejl K. Factors determining sleep in patients hospitalised in ICUs in a hospital in Northern Poland. Sleep Biol Rhythms 2019; 17: 243-50.

15. Tramontano M, De Angelis S, Galeoto G, Cucinotta MC, Lisi D, Botta RM, et al. Physical Therapy Exercises for Sleep Disorders in a Rehabilitation Setting for Neurological Patients: A Systematic Review and Meta-Analysis. Brain Sci 2021; 11: 1176.

16. Palagini L, Ragno G, Caccavale L, Gronchi A, Terzaghi M, Mauri M, et al. Italian validation of the Sleep Condition Indicator: A clinical screening tool to evaluate Insomnia Disorder according to DSM-5 criteria. Int J Psychophysiol 2015; 98 (3 Pt 1): 435-40.

17. Espie CA, Kyle SD, Hames P, Gardani M, Fleming L, Cape J. The Sleep Condition Indicator: A clinical screening tool to evaluate insomnia disorder. BMJ Open 2014; 4: e004183.

18. Espie CA, Farias Machado P, Carl JR, Kyle SD, Cape J, Siriwardena AN, et al. The Sleep Condition Indicator: Reference values derived from a sample of 200000 adults. J Sleep Res 2018; 27(3): e12643.

19. Galeoto G, Mollica R, Astorino O, Cecchi R. Il consenso informato in fisioterapia: Proposta di una modulistica. G Ital Med Lav Ergon 2015; 37: 245-54. 
20. Galeoto G, De Santis R, Marcolini A, Cinelli A, Cecchi R. The informed consent in Occupational Therapy: Proposal of forms. G Ital Med Lav Ergon 2016; 38: 107-15.

21. Galeoto G, Scialpi A, Grassi ML, Berardi A, Valente D, Tofani M, et al. General Sleep Disturbance Scale: Translation, cultural adaptation, and psychometric properties of the Italian version. CRANIO® 2021; 39(4): 326-334.

22. Panuccio F, Galeoto G, Marquez MA, Grassi ML, Scialpi A, Tofani M, et al. General Sleep Disturbance Scale (GSDS-IT) in people with spinal cord injury: A psychometric study. Spinal Cord 2020; 58(11): 1183-1188.

23. Mahoney FI, Barthel DW. Functional evaluation: The Barthel Index. Md State Med J 1965; 14: 61-5.

24. Galeoto G, Lauta A, Palumbo A, Castiglia S, Mollica R, Santilli V, et al. The Barthel Index: Italian Translation, Adaptation and Validation. Int J Neurol Neurother 2015, 2: 2.

25. Castiglia SF, Galeoto G, Lauta A, Palumbo A, Tirinelli F, Viselli F, et al. The culturally adapted Italian version of the Barthel Index (IcaBI): Assessment of structural validity, inter-rater reliability and responsiveness to clinically relevant improvements in patients admit- ted to inpatient rehabilitation centers. Funct Neurol 2017; 22: 221-8.

26. Hoch CC, Reynolds CF, Jennings JR, Monk TH, Buysse DJ, Machen MA, et al. Daytime sleepiness and performance among healthy 80 and 20 year olds. Neurobiol Aging 1992; 13(2): 353-6.

27. Stenholm S, Kronholm E, Sainio P, Borodulin K, Era P, Fogelholm M, et al. Sleep-related factors and mobility in older men and women. Journals Gerontol Ser A Biol Sci Med Sci 2010; 65A(6): 649-657.

28. Gooneratne NS, Weaver TE, Cater JR, Pack FM, Arner HM, Greenberg AS, et al. Functional outcomes of excessive daytime sleepiness in older adults. J Am Geriatr Soc 2003; 51(5): 642-9.

29. Tamrat R, Huynh-Le MP, Goyal M. Non-pharmacologic interventions to improve the sleep of hospitalized patients: A systematic review. J Gen Intern Med 2014; 29(5): 788-95.

30. Sandoval CP. Nonpharmacological interventions for sleep promotion in the intensive care unit. Crit Care Nurse 2015; 2015(10): CD008808.

31. Miller MA, Renn BN, Chu F, Torrence N. Sleepless in the hospital: A systematic review of non-pharmacological sleep interventions. Gen Hosp Psychiatry 2019; 59: 58-66. 